\title{
Ámbito de Hogar de la lagartija de las lomas Microlophus tigris (Sauria: Tropiduridae) en las Lomas de Carabayllo, Lima - Perú
}

\author{
Home Range of Lomas Lizard Microlophus tigris (Sauria: Tropiduridae) in Carabayllo Lomas, \\ Lima - Peru
}

\author{
Jorge Ruiz S. ${ }^{1}$, Karina Junes ${ }^{1}$ y José Pérez Z. ${ }^{1,2}$
}

\author{
1 Laboratorio de Estudios en Biodiversidad, Departamento de Ciencias Biológicas y Fisiológicas, Facultad de Ciencias y Filosofía, Universidad Peruana \\ Cayetano Heredia, Perú. \\ 2 Departamento de Herpetología, Museo de Historia Natural, Universidad Nacional Mayor de San Marcos, Perú. \\ Email Jorge Ruiz: ruizs.jorge@gmail.com \\ Email Karina Junes: karinajunes@gmail.com \\ Email José Pérez: jose.perez.z@upch.pe
}

\begin{abstract}
Resumen
El ámbito de hogar o "home range", es el área donde un individuo encuentra todos los recursos para su supervivencia, por ende su estudio es fundamental para conocer su ecología y estado de conservación. La lagartija de las Lomas, Microlophus tigris, es una especie endémica de la costa central del Perú, cuyo ámbito de hogar no ha sido estudiado. Para estudiar el uso del espacio de $M$. tigris se evaluó el ámbito de hogar de 15 individuos (9 machos y 6 hembras) durante la época no reproductiva en las Lomas de Carabayllo, Lima, Perú. Se empleó el método del Mínimo Polígono Convexo para estimar el área del ámbito de hogar. Los machos fueron significativamente más grandes y pesados que las hembras. No obstante, no hubo diferencias significativas entre el ámbito de hogar de los machos y hembras debido a la variabilidad del ámbito de hogar de los machos. El tamaño del ámbito de hogar de las lagartijas evaluadas no estuvo relacionado con su tamaño corporal (Longitud hocico-cloaca) ni con su peso. La ausencia de relación entre el ámbito de hogar y las variables analizadas sugiere que el tamaño del ámbito de hogar de estos reptiles, durante la época no reproductiva, respondería a otros parámetros como la abundancia de recursos, condiciones climáticas, entre otros. La baja superposición del ámbito de hogar intra e intergéneros, indicaría una evidencia indirecta de comportamiento territorial. A partir de nuestros datos y antecedentes en otras especies de saurios, consideramos que puede haber variaciones en el uso del espacio durante otras épocas del año, por lo tanto se necesitan evaluaciones complementarias a lo largo del año para observar los otros factores que determinan el tamaño del ámbito de hogar de $M$. tigris.
\end{abstract}

Palabras clave: home range; saurio; desierto costero; Lomas.

\section{Abstract}

The home range is the area where an individual finds the resources for its survival; therefore, the home range studies of organisms are fundamental to know their ecology and conservation status. The Loma's lizard, Microlophus tigris is an endemic species of Peruvian central coast, whose home range has not been studied. We used the Minimum Convex Polygon method on 15 individuals ( 9 males and 6 females) to estimate the home range of $M$. tigris during the non-breeding season in Lomas de Carabayllo, Lima, Peru. We found males were significantly larger and heavier than females. However, there were no significant differences between male and female home ranges due to high variability in males. The home range size of the evaluated lizards was not related to their body size (snout vent-length) or their weight. The lack of relation between home range and the analyzed variables suggests that the home range size of $M$. tigris, during the non-breeding season, may be related to other parameters such as resource availability or climatic conditions. The low home range overlap between and among sexes, indirectly indicates there is territorial behavior. According to our data and natural history information of other species of lizards, we believe there may be variation in the spatial use during other seasons. Therefore, it is necessary complementary evaluations to observe other relevant factors that define home range of $M$. tigris.

Keywords: home range; lizard; coastal desert; Lomas.

Citación:

Ruiz S. J., K. Junes y J. Pérez Z. 2017. Ámbito de Hogar de la Lagartija de las Lomas Microlophus tigris (Sauria: Tropiduridae) en las Lomas de Carabayllo, Lima - Perú. Revista peruana de biología 24(4): 357 - 362 (diciembre 2017). doi: http://dx.doi.org/10.15381/rpb.v24i4.14070

$\begin{array}{ll}\text { Presentado: } & 13 / 02 / 2017 \\ \text { Aceptado: } & 18 / 12 / 2017\end{array}$

online:
Información sobre los autores:

JRS realizó las evaluaciones de campo, análisis de los datos y elaboró el presente artículo. KJ realizó las evaluaciones de campo, análisis de los datos y elaboró el presente artículo. JPZ participó en las evaluaciones de campo, y elaboró el presente artículo. Todos los autores revisaron y aprobaron el manuscrito.

Los autores no incurren en conflictos de intereses. 


\section{Introducción}

El ámbito de hogar $(\mathrm{AH})$, área de vida, dominio vital o "home range", es el área total en la cual se mueve un individuo y desarrolla su ciclo de vida (Rose 1982), ya que en esta área se satisfacen todos los requerimientos para su supervivencia tales como agua, alimento, pareja, refugio y condiciones climáticas adecuadas (Christian \& Waldschmidt 1984, Wone \& Beauchamp 2003). El tamaño del AH varía considerablemente entre las especies de lagartijas, afectadas por algunos factores ecológicos y depende de variables temporales, energéticas, sexuales y reproductivas (Turner et al. 1969, Schoener 1981, Rose 1982, Christian \& Waldschmidt 1984). Algunos estudios muestran que el AH de una lagartija puede estar influenciado por el tamańo del animal o por su nivel trófico (Christian \& Waldschmidt 1984, Van Sluys 1997, Rocha 1999). De esta manera, el AH refleja varios aspectos del comportamiento de las especies (Ferner 1974) y su tamaño es un indicador de los requerimientos de recursos en relación a su disponibilidad en el ambiente, de modo que existe un interés constante en ecología por comprender los factores que predicen las características del AH (Perry \& Garland 2002).

Aunque se han realizado estudios sobre el AH en lagartos de Sudamérica en los géneros Liolaemus (7 especies), Tropidurus (6 especies), Phymaturus (2 especies), Tupinambis (2 especies) y Conolophus (1 especie), podemos considerarlos escasos y que aún no existe una comprensión general de los patrones y procesos que dan forma a la utilización del espacio por parte de los lagartos Sudamericanos (Passos et. al. 2015).

La lagartija de las Lomas, Microlophus tigris Tschudi (1845), es endémica del Desierto Costero Peruano y es el reptil más frecuente en las Lomas de la costa central peruana (Dixon \& Wright 1975, Carrillo \& Icochea 1995). Esta especie presenta una dieta generalista, un modo de forrajeo activo (Pérez 2005, Pérez et al. 2008), y se sabe que la época reproductiva de las hembras ocurre entre los meses de setiembre a diciembre (Dixon \& Wrigth 1975, Goldberg 2008). Además, es considerada como una especie Casi Amenazada por la legislación peruana (MINAGRI 2014). A pesar de ser una especie de amplia distribución en Perú y, pese a que su conservación está priorizada por el Estado Peruano, no se conocen las características básicas del $\mathrm{AH}$ de $M$. tigris en ninguna de sus poblaciones.

El objetivo de este trabajo es i) estimar el tamaño del AH de $M$. tigris en las Lomas de Carabayllo durante la época no reproductiva, ii) determinar si existen diferencias sexuales en el AH de $M$. tigris y iii) evaluar la proporción de superposición entre los $\mathrm{AH}$ de individuos adultos. De esta manera, intentamos contribuir con información sobre el AH de una especie perteneciente a un ecosistema frágil como las Lomas Costeras (MINAM 2006), en una zona sometida a la presión del crecimiento urbano de la ciudad de Lima.

\section{Materiales y métodos}

El estudio se realizó en las Lomas de Carabayllo (1148'28”S, $\left.77^{\circ} 02^{\prime} 32^{\prime \prime} \mathrm{W}\right)$, ubicada al norte de la provincia de Lima, departamento de Lima, Perú, perteneciente al ecosistema de Lomas costeras. Este ecosistema costero se caracteriza por la presencia de formaciones vegetales marcadamente estacionales producidas por la interceptación de neblina en las zonas colinosas de pendiente abrupta, presentes en el Desierto Costero (Rundel et al. 1991). Las Lomas de Carabayllo presentan suelos arenosos en las partes bajas y arenosos-arcillosos en las partes intermedias y altas, con abundantes zonas pedregosas y afloramientos rocosos, y cuya altitud se encuentra entre los 500 y $907 \mathrm{~m}$ de altitud (Cano et al. 2001).

Treinta individuos adultos (17 machos y 13 hembras) de $M$. tigris fueron capturados durante la época no reproductiva del 2012, en los meses de enero y febrero. La época reproductiva de $M$. tigris se encuentra entre los meses de setiembre a diciembre (Dixon \& Wright 1975, Goldberg 2008). Se registró la longitud hocico-cloaca (LHC) con un calibrador ( $0.1 \mathrm{~mm}$ de precisión), y la masa con una balanza de campo ( $0.1 \mathrm{~g}$ de precisión).

Los individuos fueron clasificados como adultos si tuvieron un tamaño mínimo de $50 \mathrm{~mm}$ y presentaron los patrones de coloración característicos de machos o hembras adultos (Dixon \& Wright 1975), con lo cual se registró el sexo de las lagartijas.

Se marcaron los individuos mediante corte de falanges siguiendo la codificación de Pérez (2005). Adicionalmente, se realizaron marcas laterales temporales con esmalte de uso cosmético no tóxico, para hacer posible la identificación a la distancia. Se empleó el color negro debido a que es poco llamativo y no afecta el camuflaje natural del diseño corporal de este saurio. Posteriormente al marcaje, las lagartijas fueron liberadas en el mismo lugar de su captura. Cada registro y recaptura de las lagartijas fue ubicado en un plano cartesiano con ayuda de una brújula y cinta métrica. La toma de datos se realizó cada dos días entre las 09:00 y 17:00 h, coincidiendo con el horario de actividad de la especie (Pérez 2005). Se registró un avistamiento por individuo por día para evitar la autocorrelación de los datos, excepto en dos ocasiones, donde se realizaron dos avistamientos en un mismo día, para maximizar la cantidad de registros, y debido a la baja probabilidad de relación entre estos registros.

Se evaluó la normalidad y heterocedasticidad de los datos para determinar el uso de pruebas paramétricas y no paramétricas, según corresponda. Se realizaron comparaciones del tamaño y peso entre sexos mediante la prueba Mann-Whitney (Zar 1999).

El AH se estimó con el método del Mínimo Polígono Convexo (MPC) (Rose 1982), para lo cual se empleó el programa Biotas 2.0 Alpha (Biotas 2004). Con este mismo programa se estimó el área superpuesta entre los $\mathrm{AH}$ de los individuos y se hicieron comparaciones mediante la prueba T-student (Zar 1999). El MPC proporciona el área delimitada por los contornos que encierran todos los avistamientos de una lagartija y no asume alguna hipótesis a priori sobre la distribución espacial de los individuos (Haenel et. al. 2003), por lo tanto se requiere como mínimo tres registros de los individuos para estimar el $\mathrm{AH}$. En este trabajo, obtuvimos los registros necesarios para estimar el AH de nueve machos y seis hembras de $M$. tigris, de los 30 individuos que logramos marcar.

Se comparó el AH entre machos y hembras mediante la prueba de Mann-Whitney (Zar 1999). Mediante una regresión lineal (Zar 1999) se evaluó la relación entre el AH con la LHC, y el AH con el peso de las lagartijas. Adicionalmente, siguiendo la metodología propuesta por Rose (1982) para determinar el número mínimo de avistamientos para estimar el $\mathrm{AH}$, se relacionó el número de avistamientos y el porcentaje del área acumulada del AH ajustándolos a una regresión logarítmica. Todas las pruebas estadísticas se realizaron con el programa PAST 3.0 (Hammer et al. 2001) con un nivel de significancia establecido en $\alpha=0.05$. 


\section{Resultados}

La LHC promedio de los machos $(80.1 \mathrm{~mm}$, IC 95\% = 76.4 $-83.8 \mathrm{~mm}, \mathrm{n}=16)$ fue mayor que en las hembras $(65.4 \mathrm{~mm}$, IC $95 \%=63.4-67.4 \mathrm{~mm}, \mathrm{n}=12)($ Test $\mathrm{U}=8.0, \mathrm{p}<0.01)$. Del mismo modo, el peso promedio de los machos (17.5 g, IC 95\% $=15.2-19.8 \mathrm{~g}, \mathrm{n}=16$ ) fue mayor que en las hembras (8.6 g, IC $95 \%=7.6-9.6 \mathrm{~g}, \mathrm{n}=12$ ) (Test $\mathrm{U}=8.0 \mathrm{p}<0.01)$, presentando una alta relación entre la LHC y el peso $\left(\mathrm{R}^{2}=0.9, \mathrm{p}<0.01\right)$.

El tamaño promedio del AH de $M$. tigris fue de $122.03 \mathrm{~m}^{2}$ (IC $95 \%=48.9-195.1 \mathrm{~m}^{2}, \mathrm{n}=15$ ). El tamaño del AH de las lagartijas evaluadas no se encuentra relacionada con su tamaño corporal $\left(\mathrm{R}^{2}=0.096, \mathrm{p}=0.3, \mathrm{n}=13\right)$, ni con su peso $\left(\mathrm{R}^{2}=0.11\right.$, $\mathrm{p}=0.26, \mathrm{n}=13)$. No se encontraron diferencias entre el tamańo promedio del AH de machos $\left(163.2 \mathrm{~m}^{2}\right.$, IC 95\% = 49.1 - 277.2 $\left.\mathrm{m}^{2}, \mathrm{n}=9\right)$ y de hembras $\left(60.2 \mathrm{~m}^{2}\right.$, IC $95 \%=25.3-95.1 \mathrm{~m}^{2}$, $\mathrm{n}=6$ ) (Test $\mathrm{U}=15.0, \mathrm{p}=0.18$ ) (Fig. 1). Sin embargo, la varianza del $\mathrm{AH}$ de los machos fue mayor que en el caso de las hembras (Test Levene: $\mathrm{p}<0.01$ ) (Fig. 1).

La superposición del AH de los machos ocurrió en una sola ocasión, siendo esta en promedio el $44.3 \%(n=2)$ del AH de ambos individuos (Fig. 2), mientras que entre las hembras no se registró superposición de sus $\mathrm{AH}$. En todos los casos en los que se registró superposición de las áreas entre machos y hembras, el porcentaje del área de AH superpuesta de las hembras (86.2\%, $\mathrm{n}=3$ ) sobre el área de $\mathrm{AH}$ de los machos $(21.7 \%, \mathrm{n}=3)$ fue mayor (Test $\mathrm{t}=3.33, \mathrm{p}=0.03)$ (Fig. 2). La curva logarítmica $(\mathrm{y}$ $\left.=61.6 \ln (\mathrm{x})-14.814 ; \mathrm{R}^{2}=0.645\right)$ mostró su máximo valor de $\mathrm{AH}(100 \%)$ al realizarse ocho avistamientos y mostró un AH de $80 \%$ con cinco avistamientos, sin embargo no se observó una aproximación a la asíntota (Fig. 3).

\section{Discusión}

Microlophus tigris presenta dimorfismo sexual en la LHC, como lo reportado en otras especies de Tropidúridos (Schoener et. al. 1982, Pérez-Mellado \& De la Riva 1993, Vidal et. al. 2002, Pinto et. al. 2005), siendo los machos más grandes que las hembras. Esto también se refleja en el peso, pues los machos pesaron más que las hembras. Al igual que en Tropidurus tor-

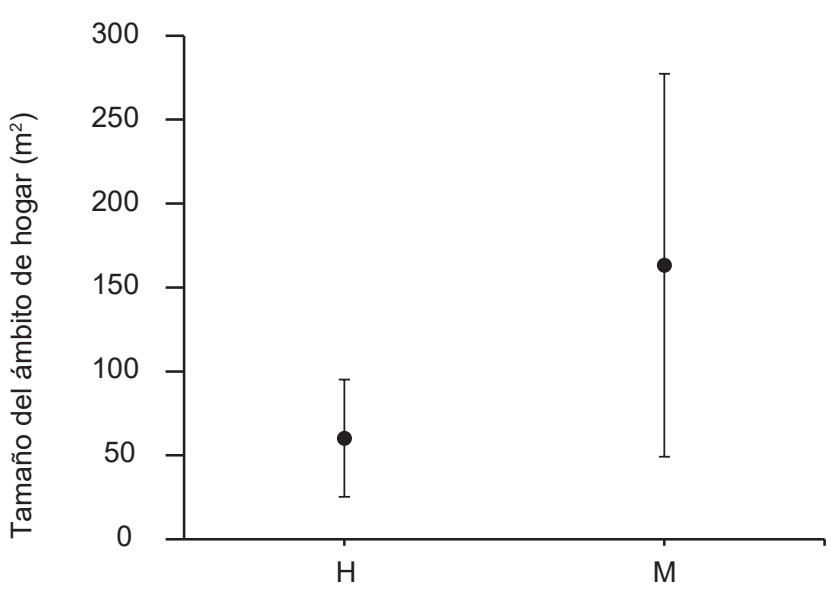

Figura 1. Tamaño del Ámbito de Hogar (IC 95\%) de machos (M) y hembras $(\mathrm{H})$ de Microlophus tigris durante la época no reproductiva en las Lomas de Carabayllo.

quatus (Ribeiro et al. 2009), no se encuentra relación entre estas variables con el $\mathrm{AH}$, mientras que en T. itambere (Van Sluys 1997) el tamaño del AH incrementa con la LHC y el peso. Con frecuencia se asume que animales más grandes requieren mayores AH para satisfacer sus requerimientos energéticos (Turner et al. 1969), esperando una relación entre la LHC y el peso con el AH. Sin embargo, mayores tamańos de $\mathrm{AH}$ implican un mayor costo metabólico debido a un mayor desplazamiento (Rose 1982). Esto indicaría que en $M$. tigris el tamaño del AH varía independientemente de la LHC y el peso, y que esta relación responde a factores sociales, cambios estacionales o variaciones en la productividad del hábitat (Christian \& Waldschmidt 1984).

En esta investigación se empleó la metodología estándar para evaluar el AH de lagartijas, por lo tanto, son posibles las comparaciones con otros estudios. El tamaño promedio del $\mathrm{AH}$ de individuos machos y hembras de $M$. tigris es mayor comparado con otros Tropidúridos (Tabla 1), como T. itambere (Van Sluys 1997) y T. torquatus (Giaretta 1996, Ribeiro et al. 2009); y algunos Liolaemidos como Liolaemus lutzae (Rocha 1999), L.

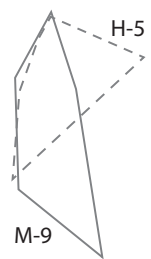

$5 \mathrm{~m}$
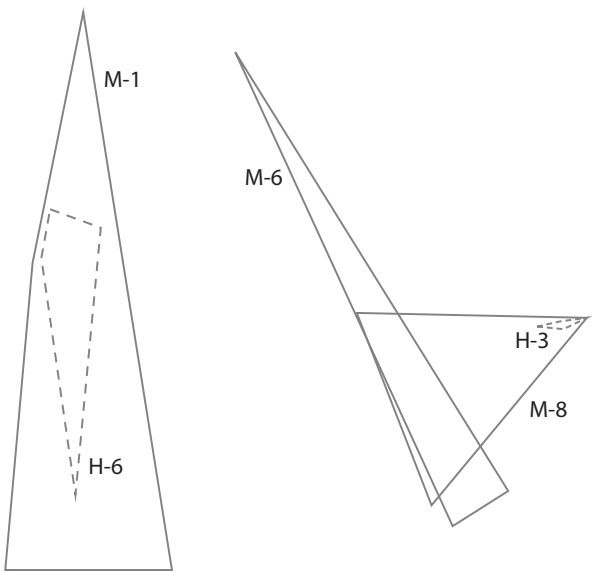

Figura 2. Superposición del Ámbito de Hogar $(\mathrm{AH})$ de machos (M) y hembras $(\mathrm{H})$ de Microlophus tigris durante la época no reproductiva en las Lomas de Carabayllo. Los números indican el individuo estudiado. Líneas punteadas corresponden al $\mathrm{AH}$ de las hembras.

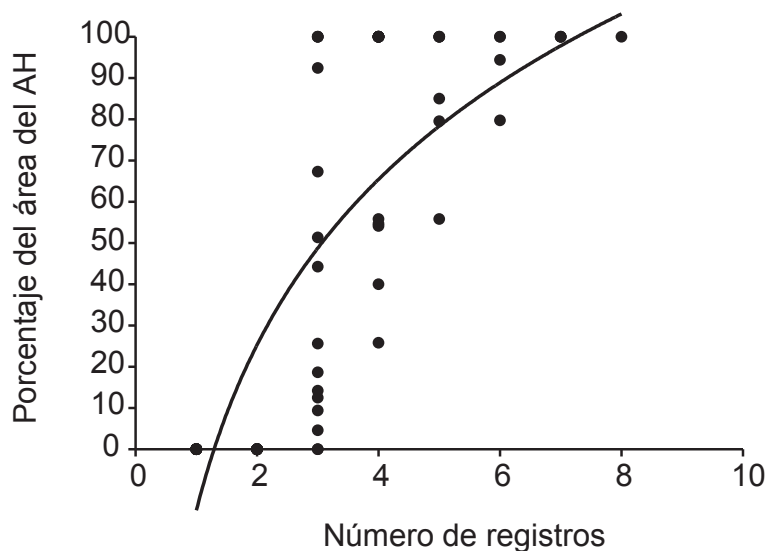

Figura 3. Porcentajes acumulados del área del Ámbito de Hogar de Microlophus tigris en las Lomas de Carabayllo. 
quilmes (Halloy \& Robles 2002), L. koslowskyi (Frutos \& Belver 2007), L. melanops (Frutos et. al. 2007) y L. multimaculatus (Kacorilis et. al. 2009) (Tabla 1). Estas diferencias son importantes considerando que las especies mencionadas presentan tamaños corporales similares al de $M$. tigris (Tabla 1). Estas diferencias pueden ser atribuidas al tipo de dieta y modo de forrajeo de $M$. tigris y las especies mencionadas (Tabla 2), como lo propone Rose (1982) para otros saurios, señalando que las especies de saurios de forrajeo activo usualmente presentan $\mathrm{AH}$ más grandes que las especies de forrajeo pasivo. Por otro lado, Perry \& Garland (2002) sugieren que los carnívoros (insectívoros) presentan tamaños de $\mathrm{AH}$ más grandes con respecto a los omnívoros y herbívoros. Microlophus tigris es una especie insectívora generalista (Pérez 2005) de forrajeo activo (Pérez et. al. 2008), lo cual justificaría el mayor AH frente a las del género Tropidurus y Liolaemus que presentan forrajeo pasivo y dietas insectívorasomnívoras (Tabla 2).

Cabe mencionar que otra posible explicación a las diferencias indicadas en el tamaño del $\mathrm{AH}$ entre $M$. tigris y los otros Tropidúridos, sería las diferencias en las abundancias de las presas en el hábitat. El Desierto Costero Peruano presenta bajas abundancias de invertebrados (Aguilar 1985), comparado con los desiertos menos áridos. Las abundancias de recursos alimentarios son un factor muy importante en el modelamiento de las formas y tamaños del AH de saurios (Rose 1982). Para el caso de $M$. tigris, inclusive se ha reportado un consumo limitado de material vegetal, que podría ser un potencial suplemento en la dieta (Pérez 2005), sin embargo, este recurso es escaso en las Lomas costeras peruanas en épocas de verano (diciembre a marzo), en el momento del desarrollo de esta evaluación.

En nuestro estudio, la ausencia de diferencias entre los tamaños promedios de $\mathrm{AH}$ de machos y hembras de $M$. tigris se debe a la mayor variabilidad del $\mathrm{AH}$ de los machos. La variabilidad del AH de los machos indicaría la probabilidad de recorrer áreas más extensas y por ende presentar mayores tamańos de $\mathrm{AH}$, que podría estar influenciado por factores como la agresividad, la dominancia de algunos machos sobre otros y la búsqueda de recursos. La disponibilidad de hembras también determina el tamaño del AH de los saurios machos, ya que esto asegura su éxito reproductivo (Perry \& Garland 2002).

Por otro lado, la ausencia de diferencias entre los tamaños de $\mathrm{AH}$ de machos y hembras también puede estar influenciada por

Tabla 2. Tipo de dieta y estrategia de forrajeo de Microlophus tigris y otras especies de lagartijas sudamericanas. A: Forrajeo activo. SW: Forrajeo pasivo. ni: no indica.

\begin{tabular}{llcl}
\hline \multicolumn{1}{c}{ Especie } & \multicolumn{1}{c}{ Dieta } & $\begin{array}{c}\text { Estrategia de } \\
\text { Forrajeo }\end{array}$ & \multicolumn{1}{c}{ Referencia bibliográfica } \\
\hline Microlophus tigris & Insectívoro - generalista & $\mathrm{A}$ & Pérez 2005, Pérez et al. 2008 \\
Tropidurus itambere & Omnívoro & $\mathrm{SW}$ & Van Sluys 1993 \\
Tropidurus torquatus & Generalista & $\mathrm{SW}$ & Teixeira \& Giovanelli 1999, Siqueira et. al. 2013 \\
Liolaemus koslowskyi & Generalista & $\mathrm{ni}$ & Belver \& Ávila 2000 \\
Liolaemus lutzae & Omnívoro, adultos herbívoros & $\mathrm{SW}$ & Rocha 1996, Rocha 1998 \\
Liolaemus melanops & Insectívoro & $\mathrm{ni}$ & Frutos et al. 2007 \\
Liolaemus multimaculatus & Insectívoro & $\mathrm{SW}$ & Kacorilis et. al. 2009 \\
Liolaemus quilmes & Insectívoro & $\mathrm{ni}$ & Halloy et al. 2006 \\
\hline
\end{tabular}

Tabla 1. Tamaño promedio del Ámbito de Hogar $\left(\mathrm{AH}, \mathrm{m}^{2}\right)$ y Longitud hocico-cloaca (LHC, $\left.\mathrm{mm}\right)$ de Microlophus tigris y otras especies de lagartijas sudamericanas de las familias Tropiduridae y Liolaemidae durante la época no reproductiva. SD: desviación estándar. ( ${ }^{*}$ ) Datos de la época reproductiva. ( $\left.{ }^{a}\right)$ Ámbito de Hogar de dos años. ( $\left.{ }^{b}\right)$ Longitud hocico-cloaca de dos años.

\begin{tabular}{|c|c|c|c|c|c|c|c|c|}
\hline Especies & & Machos & & SD & Hembras & & SD & Referencia bibliográfica \\
\hline \multirow[t]{2}{*}{ Microlophus tigris } & $\mathrm{AH}$ & 163.2 & \pm & 174.6 & 60.2 & \pm & 43.6 & en este estudio \\
\hline & LHC & 80.1 & \pm & 7.5 & 65.2 & \pm & 3.4 & \\
\hline \multirow[t]{3}{*}{ Tropidurus itambere } & $\mathrm{AH}$ & 104.9 & \pm & 107.11 & 15.5 & \pm & 19.04 & Van Sluys 1997a \\
\hline & $\mathrm{AH}$ & 28.1 & \pm & 26.7 & 11.9 & \pm & 13.4 & \\
\hline & LHC & 75.9 & \pm & 11.8 & 67.3 & \pm & 7.2 & Van Sluys 1993 \\
\hline \multirow[t]{3}{*}{ Tropidurus torquatus } & $\mathrm{AH}$ & 72.6 & \pm & 32.9 & 58.9 & \pm & 21.8 & Ribeiro et. al. 2009 \\
\hline & $\mathrm{AH}$ & 57.9 & \pm & 18 & 11.8 & \pm & 11.4 & Giaretta 1996 \\
\hline & LHC & 71.6 & \pm & 3.7 & 58.2 & \pm & 2.6 & \\
\hline \multirow[t]{3}{*}{ Liolaemus koslowskyi } & $\mathrm{AH}$ & 139.7 & \pm & 135.23 & 35.5 & \pm & 34.8 & Frutos \& Belver 2007b \\
\hline & LHC & 59.3 & \pm & 7.2 & 55.6 & \pm & 7 & \\
\hline & LHC & 57.8 & \pm & 6.52 & 55.62 & \pm & 6.04 & \\
\hline \multirow[t]{2}{*}{ Liolaemus lutzae } & $\mathrm{AH}^{*}$ & 59.8 & \pm & 33.7 & 22.3 & \pm & 16.1 & Rocha 1999 \\
\hline & LHC & 78 & & & 62 & & & Rocha 1992 \\
\hline \multirow[t]{2}{*}{ Liolaemus melanops } & $\mathrm{AH}$ & 70.9 & \pm & 37.4 & 42.1 & \pm & 51.3 & Frutos et. al. 2007 \\
\hline & LHC & 76.4 & \pm & 0.64 & 71.8 & \pm & 0.52 & \\
\hline \multirow[t]{2}{*}{ Liolaemus multimaculatus } & $\mathrm{AH}$ & 39.87 & \pm & 79.57 & 54.96 & \pm & 66.41 & Kacorilis et. al. 2009 \\
\hline & LHC & $47-56$ & & & $50-64$ & & & \\
\hline \multirow[t]{4}{*}{ Liolaemus quilmes } & $\mathrm{AH}$ & 132.2 & \pm & 82.7 & 29.2 & \pm & 9.5 & Halloy \& Robles 2002 \\
\hline & $\mathrm{AH}^{*}$ & 180 & \pm & 71 & 31 & \pm & 17 & Robles \& Halloy 2009ª \\
\hline & $\mathrm{AH}$ & 143 & \pm & 69 & 32 & \pm & 20 & \\
\hline & LHC & 61.3 & \pm & 0.48 & 57.2 & \pm & 0.37 & Halloy \& Robles 2002 \\
\hline
\end{tabular}


la época del año. En T. torquatus, Ribeiro et al. (2009) no encuentran diferencias entre los AH de machos y hembras durante la época no reproductiva, mientras que en la época reproductiva el tamaño del AH de los machos fue mayor que para las hembras. Stamps (1983) reporta que en algunos saurios, el tamaño del $\mathrm{AH}$ de los machos es entre 3.5 a 4 veces más grande que las hembras durante la época reproductiva, mientras que en la época no reproductiva, el tamaño del $\mathrm{AH}$ no difiere entre sexos. Por lo tanto, resulta necesario realizar estudios de $\mathrm{AH}$ durante la época reproductiva de $M$. tigris, para poder determinar el probable incremento del $\mathrm{AH}$ en esta época y poder conocer el desarrollo de esta especie a lo largo de todo año.

Los machos de $M$. tigris, que tenían superposición de sus $\mathrm{AH}$ con alguna de las hembras, presentaron los mayores tamaños de $\mathrm{AH}$ registrados, indicando que posiblemente estos AH pertenecerían a machos dominantes, que probablemente tienen la capacidad de asegurar al menos una hembra para la reproducción, pues las lagartijas dominantes controlan $\mathrm{AH}$ más grandes y favorables para el apareamiento (Garland et al. 1990). La dominancia también está relacionada con el tamaño corporal, entre otros factores (Tokarz 1985), por lo tanto, es posible sugerir la dominancia de estos dos machos que presentaron las mayores LHC (88.5 mm y $83.6 \mathrm{~mm})$. Por otro lado, el tercer macho con $\mathrm{AH}$ superpuesto al de una hembra, se encontró en otra zona de la loma y presentó una LHC por debajo del promedio (LHC= $75.7 \mathrm{~mm}$ ).

Además, en los tres casos en que se registró superposición entre los $\mathrm{AH}$ de machos y hembras, el AH de los machos solo incluía una hembra. A diferencia de este hallazgo, los machos de T. itambere (Van Sluys 1997) y T. torquatus (Ribeiro et al. 2009) presentan, en promedio, superposición de sus AH hasta con tres hembras y este número se incrementa durante la época reproductiva en ambas especies (Van Sluys 1997, Ribeiro et al. 2009). Se ha reportado que el éxito de apareamiento de los saurios machos aumenta a medida que se incrementa el número de hembras dentro de su $\mathrm{AH}$, y disminuye cuando otros machos superponen sus AH con las mismas hembras (Rose 1982). Otra forma de asegurar el éxito reproductivo sería resguardar a una hembra para que esta no se aparee con otros machos, lo que se convierte en una relación monogámica, al menos temporal (Bull 2000). Por lo tanto, el AH de los individuos machos de $M$. tigris podría incrementarse durante la época reproductiva abarcando más hembras o podría mantenerse indicando la monogamia de esta especie. No se cuenta con información sobre el sistema de apareamiento en $M$. tigris, por lo que sugerimos evaluar ambas posibilidades en estudios posteriores.

El bajo porcentaje de superposición entre machos de $M$. tigris, sugiere una exclusión mutua y uso de áreas exclusivas (Ribeiro et al. 2009), como en T. itambare (Van Sluys 1997), T. torquatus (Ribeiro et al. 2009) y L. lutzae (Rocha 1999), que indicaría un comportamiento territorial (Rose 1982). Por otro lado, la nula superposición entre los AH de las hembras de $M$. tigris, es similar con lo registrado en L. lutzae (Rocha 1999), L. quilmes (Halloy \& Robles 2002), L. koslowskyi (Frutos \& Belver 2007) y L. melanops (Frutos et. al. 2007). En contraste, las hembras de T. itambere (Van Sluys 1997) y T. torquatus (Ribeiro et al. 2009) presentan superposición en sus AH durante la época reproductiva y la no reproductiva. Es posible que nuestro registro se deba a lo mencionado por Rose (1982), quien señala que la frecuencia de encuentros entre hembras es baja debido a que tienen $\mathrm{AH}$ menores, en comparación a los machos, y sus distancias de movimiento diario son cortas. Esto implica que la superposición de los $\mathrm{AH}$ de las lagartijas hembras sea poco frecuente.

La curva de acumulación del tamaño del AH sugiere que para nuestro estudio se requiere como mínimo cinco avistamientos por individuo para obtener el $80 \%$ del área del AH de $M$. tigris y al menos ocho avistamientos para obtener el 100\% del AH, asumiendo que hemos estimado el tamaño real total del $\mathrm{AH}$ de M. tigris. De igual manera, Ribeiro et al. (2009) reportan que la relación entre el tamaño promedio del $\mathrm{AH}$ de $T$. torquatus y el número de avistamientos se estabiliza después de ocho avistamientos. Por lo tanto, se proporciona un estimado del esfuerzo mínimo de muestreo para evaluar el tamaño del $\mathrm{AH}$ de Tropidúridos del Desierto Costero Peruano.

Considerando estos antecedentes y lo registrado en nuestro trabajo, sugerimos que $M$. tigris tiene un comportamiento territorial, y es posible que en la época reproductiva se presente una diferencia importante entre los $\mathrm{AH}$ de machos y hembras de $M$. tigris, siguiendo el patrón general de las lagartijas, donde los machos tienen un AH mayor al de las hembras (Rose 1982, Giaretta 1996).

Sugerimos incluir, en futuras investigaciones, comparaciones estacionales y anuales, observaciones comportamentales y aspectos reproductivos de $M$. tigris que aporten al conocimiento de la biología de esta especie y generen información para elaborar una estrategia de conservación adecuada.

\section{Agradecimientos}

A Ciara Sánchez y Fernando Mamani por la ayuda en el trabajo de campo. A Katya Balta por la revisión de las primeras versiones del manuscrito.

\section{Literatura citada}

Aguilar P. 1985. Fauna de las Lomas costeras del Perú. Boletín de Lima 7(41): 17-28.

Belver L. C. \& L. J. Ávila. 2000. Herbivoría: Solo para grandes lagartos? XV Reunión de Comunicaciones Herpetológicas de la Asociación Herpetológica Argentina. Bariloche, Rio Negro, Octubre 2000.

Biotas $^{\mathrm{TM}}$. 2004. Ecological Software Solutions LLC. Hegymagas, Hungary. Version 2.0

Bull C.M. 2000. Monogamy in lizards. Behavioural Processes 51:7-20. doi http://dx.doi.org/10.1016/S0376-6357(00)00115-7

Cano A., M. Arakaki, J. Roque, M.I. La Torre, N. Refulio, C. Arana. 2001. Flora vascular en las lomas de Ancón y Carabayllo Lima, Perú durante El Niño 1997-98. In: Tarazona J., Arntz W.E., Castillo de Maruenda E., editors. El Niño en América Latina: Impactos Biológicos y Sociales. Lima: Consejo Nacional de Ciencia y Tecnología. pp. 259-265

Carrillo N. \& J. Icochea. 1995. Lista taxonómica preliminar de los Reptiles vivientes del Perú. Publicaciones del Museo de Historia Natural UNMSM. Serie A: Zoología. 49: 1-27.

Christian K.A. \& S. Waldschmidt. 1984. The relationship between lizards Home Range and body size: A reanalysis of the data. Herpetologica 40: 68-75.

Dixon J. \& R. Huey. 1970. Systematic of the lizards of the gekkonidae genus Phyllodactylus of mainland South America. Contribution in Science The Natural History Museum of Los Angeles. 192: 1-78.

Dixon, J. R. \& J. W. Wright. 1975. A review of the lizards of the iguanid genus Tropidurus in Peru. Natural History Museum of Los Angeles County, Contributions in Science 271:1-40 pp.

Ferner J.W. 1974. Home-range size and overlap in Sceloporus undu- 
lates erythrocheilus (Reptilia: Iguanidae). Copeia, 332-337. doi: http://dx.doi.org/10.2307/1442527

Frutos N. \& L. Belver. 2007. Dominio Vital de Liolaemus koslowskyi Etheridge, 1993 (Iguania: Liolaemini) en el Noroeste de la Provincia de la Rioja, Argentina. Cuadernos de Herpetología 21 (2): 83-92

Frutos N., Camporro L. \& A. Luciano. 2007. Ámbito de Hogar de Liolaemus melanops Burmesiter, 1888 (Squamata: Liolaemini) en el Centro de Chubut, Argentina. Gayana, Concepción, Chile. 71:142-149. doi: http://dx.doi.org/10.4067/S071765382007000200002

Garland T., E. Hankins \& R. Huey. 1990 Locomotor capacity and social dominace in male lizards. Funtional ecology 4: 243250. doi: http://dx.doi.org/10.2307/2389343

Giaretta A.A. 1996. Tropidurus torquartus (NCN). Home range. Herpetological Review 27:80-81.

Goldberg S.R. 2008. Reproduction in seven species of Microlophus (Squamata: Tropiduridae) from South America. The Texas Journal of Science. 60: 4

Haenel G.J., L.C. Smith \& H.B. John-Alder. 2003. Home range analysis in Sceloporus undulatus (Eastern Fence Lizard). I. Spacing patterns and the context of territorial behavior. Copeia. 99-112. https://doi.org/10.1643/00458511(2003)003[0099:HRAISU]2.0.CO;2

Halloy M. \& C. Robles. 2002. Spatial distribution in a neotropical lizard, Liolaemus quilmes (Liolaemidae): site fidelity and overlapping among males and females. Bulletin of Maryland Herpetological Society. 38: 118-129.

Halloy M., C. Robles \& F. Cuezzo. 2006. Diet in two syntopic neotropical lizard species of Liolaemus (Liolaemidae): interspecific and intersexual differences. Revista Española de Herpetología. 20:47-56.

Hammer O., D.A.T. Harper \& P.D. Ryan. 2001. Past: Paleontologica Statistics Software Package for education and data analysis. Paleontologia Electronica. 4:1-9.

Kacorilis F.P., J.D. Williams, C. Ruiz \& C. Cassino. 2009. Home range size and overlap in Liolaemus multimaculatus (Squamata: Liolamidae) in Pampean coastal dunes of Argentina. South American Journal of Herpetology. 4(3): 229-234. doi: http:// dx.doi.org/10.2994/057.004.0305

MINAGRI (Ministerio de Agricultura y Riego). 2014. D.S. №. 004-2014-MINAGRI. Aprueban la actualización de la lista de clasificación y categorización de las especies amenazadas de Fauna Silvestre legalmente protegidas. 08 de Abril del 2014. El Peruano Normas Legales: 520497-520504

MINAM (Ministerio del Ambiente). 2006. Ley General del Ambiente 28611. Artículo 99: Ecosistemas Frágiles. 15 de Abril del 2005.

Passos D.C., C.A.B. Galdino \& C.F.D. Rocha. 2015. Challenges and Perspectives for Studies on Home Range of Lizards from South America. South American Journal of Herpetology .10: 82-89.doi: http://dx.doi.org/10.2994/SAJH-D-14-00023.1

Perez-Mellado V. \& I. De La Riva. 1993. Sexual size dimorphism and ecology: the case of a tropical lizard, Tropidurus melanopleurus (Sauria: Tropiduridae). Copeia. 1993: 969-976. doi: http://dx.doi.org/10.2307/1447074

Pérez Z. J. 2005. Ecologia de Duas Espécies de Lagartos Simpatricos em uma Formação Vegetal de Lomas no Deserto Costeiro Peruano Central. Dissertação de Mestrado. Universidade do Estado do Rio de Janeiro (UERJ). Rio de Janeiro. Brasil.

Pérez Z. J., K. Balta, R. Ramírez \& D. Susanibar. 2008. Succinea peruviana (Gastropoda) en la dieta de la lagartija de las Lomas Microlophus tigris (Sauria) en la Reserva Nacional de Lachay, Lima, Perú. Revista Peruana de Biología. 15(1): 109-110. doi: http://dx.doi.org/10.15381/rpb.v15i1.1685

Perry G. \& T. Garland. 2002. Lizard Home Ranges revisited. Effects of sex, body size, diet, habitat and phylogeny. Ecology. 83: 1870-1885. doi: $10.2307 / 3071771$

Pinto A.C.S., H. Wiederhecker \& G.R. Colli. 2005. Sexual dimorphism in the Neotropical lizard, Tropidurus torquatus
(Squamata, Tropiduridae). Amphibia-Reptilia. 26: 127-137. doi: http://dx.doi.org/10.1163/1568538054253384

Ribeiro L.B., B.M. Souza \& S.C. Gomides. 2009. Range structure, microhabitat use, and activity patterns of the saxicolous lizard Tropidurus torquatus (Tropiduridae) on a rock outcrop in Minas Gerais, Brazil. Revista Chilena de Historia Natural. 82:577-588. doi: http://dx.doi.org/10.4067/ S0716-078X2009000400011

Robles C. \& M. Halloy. 2009. Home ranges and reproductive strategies in a neotropical lizard, Liolaemus quilmes (Iguania: Liolaemidae). South American Journal of Herpetology. 4(3): 253-258. doi: http://dx.doi.org/10.2994/057.004.0308

Rocha C. F. D. 1992. Reproductive and fat body cycles of the tropical sand lizard (Liolaemus lutzae) of Southeastern Brazil. Journal of Herpetology. 26: 17-23. doi: http://dx.doi. org/10.2307/1565016

Rocha C.F.D. 1996. Seasonal shift in lizard diet: the seasonality in food resources affecting the diet of Liolaemus lutzae (Tropiduridae). Ciência e Cultura. 48 (4): 264-269.

Rocha C.F.D. 1998, Ontogenetic shift in the rate of plant consumption in a tropical lizard (Liolaemus lutzae). Journal of Herpetology. 32(2): 274-279. doi: http://dx.doi.org/10.2307/1565309

Rocha C.F.D. 1999. Home Range of the Tropidurid lizard Liolaemus lutzae: sexual and body size differences. Revista Brasileña de Biología. 59:125-130. doi: http://dx.doi.org/10.1590/ S0034-71081999000100016

Rose B. 1982. Lizard home ranges: Methodology and functions. Journal of Herpetology. 16: 253-269. doi: http://dx.doi. org/10.2307/1563718

Rundel P. W., M.O. Dillon, B. Palma, H.A.Mooney, S.L. Gulmon \& J.R. Ehleringer. 1991. The phytogeography and ecology of the coastal Atacama and Peruvian deserts. Aliso, 13, 1-50.

Schoener T., J. Slade \& C. Stinson. 1982. Diet and sexual dimorphism in the very catholic lizard genus Leiocephalus, of the Bahamas. Oecologia 53: 160-169. doi: http://dx.doi. org/10.1007/BF00545659

Siqueira C.C., M.C. Kiefer, M. Van Sluy \& C.F.D. Rocha. 2013. Variation in the diet of the lizard Tropidurus torquatus along its coastal range in Brazil. Biota Neotropica. 13(3):93-101. doi: http://dx.doi.org/10.1590/S1676-06032013000300012

Stamps J.A. 1983. Sexual selection, sexual dimorphism, and territoriality. En: Lizard Ecology. Studies of a Model Organism. (Eds. Huey, R.B., E.R. Pianka, y T.W. Schoener). HUP, Cambridge, MA, USA.

Teixeira R.L. \& M. Giovanelli. 1999. Ecologia de Tropidurus torquatus (Sauria: Tropiduridae) da Restinga de Guriri, São Mateus, ES. Revista Brasileira de Biologia 59 (1): 11-18. doi: http:// dx.doi.org/10.1590/S0034-71081999000100002

Tokarz R. 1985. Body size as a factor determining dominance in staged agonistic encounters between male brown anoles (Anolis sagrei). Animal Behaviour 33: 746-753. doi: https://doi. org/10.1016/S0003-3472(85)80006-3

Turner F.B., R.I. Jennrich \& J.D. Weintraub. 1969. Home ranges and body sizes of Lizards. Ecology, 50: 1076-1081. doi: http:// dx.doi.org/10.2307/1936898

Van Sluys M. 1993. Food habits of the lizard Tropidurus itambere (Tropiduridae) in southeastern Brazil. Journal of Herpetology. 27(3):347-351. doi: http://dx.doi.org/10.2307/1565162

Van Sluys M. 1997. Home range of the saxicolous lizard Tropidurus itambere in Southeastern Brazil. Copeia (3): 623-628. doi: http://dx.doi.org/10.2307/1447571

Vidal M., J.C. Ortiz \& A. Labra. 2002. Sexual and age differences in ecological variables of the lizard Microlophus atacamensis (Tropiduridae) from northern Chile. Rev. chil. hist. nat. v.75 n.2. doi: http://dx.doi. org/10.4067/S0716-078X2002000200002

Wone B. \& B. Beauchamp. 2003. Movement, Home Range and activity patterns of the Horned Lizard, Phrynosoma mcallii. Journal of Herpetology, 37:679-686.

Zar J. H. 1999. Biostatistical analysis. 4 ed. Upper Saddle River. Prentice Hall. 